\title{
Digitally guided surgery and prosthesis with narrow implants in the anterior region
}

\author{
Jae-Seok Kang ${ }^{1}$, Seon-A Kim², Yu-Ri Heo ${ }^{3}$, and Mee-Kyung Son ${ }^{3 *}$ \\ ${ }^{1}$ Doctor of Dental Clinic, Department of Implant-Prosthodontics, Yedam Dental Hospital, Mokpo, Republic of Korea \\ ${ }^{2}$ Resident, Department of Prosthodontics, Collage of Dentistry, Chosun University, Gwangju, Republic of Korea \\ ${ }^{3}$ Professor, Department of Prosthodontics, Collage of Dentistry, Chosun University, Gwangju, Republic of Korea
}

The purpose of this case report is to introduce a flapless method of securely placing a narrow implant on the upper and lower anterior teeth missing area with narrow bone width using a digitally guided surgery system and to introduce a process of prosthesis manufacturing using an oral scanner without a working model. The narrow implants in this case report remained stable for a certain period of time (12, 24, and 36 months after surgery) without loss of the surrounding bones.

Key Words: Digital guided surgery; Flapless; Narrow implant; Oral scanner

(c) This is an open-access article distributed under the terms of the Creative Commons Attribution Non-Commercial License (http://creativecommons.org/licenses/by-nc/4.0) which permits unrestricted noncommercial use, distribution, and reproduction in any medium, provided the original work is properly cited.

\section{Introduction}

In general, certain amount of bone loss occurs at alveolar bone crestal area during the healing period after implantation [1,2], so it is reported that for successful osseointegration, the remaining buccal and lingual bone around the implant should be at least $2 \mathrm{~mm}$ [3]. However, since the maxillary or mandibular anterior teeth are often limited by the bucco-lingual bone width, it may be difficult to place a normal diameter implant. In this case, a method of increasing the success rate by using bone graft was recommended [4].

Mini or narrow implants were introduced for the purpose of implantation in such a narrow bone width. In general, many papers published so far have named 'mini implants' for diameters less than $3.0 \mathrm{~mm}$, and 'narrow implants' for diameters from 3.0 to $3.5 \mathrm{~mm}[5,6]$.

In many previous studies, the implant diameter has been reported as one of the most important factors for implant success. Small diameter implants have a lower success rate compared to normal diameter implants, and the critical limit of dimeter for higher success rate is often known as 3.0 $\mathrm{mm}$, and implants with a diameter of less than $3.0 \mathrm{~mm}$ are reported to have a risk of many failures [5,7].

On the other hand, a relatively high success rate has begun to be reported for implants with a diameter of $3.0 \mathrm{~mm}$ or more, and in particular, and in 2008, Zinsli et al. [8] reported a high success rate using an implant with a diameter of $3.3 \mathrm{~mm}$. In particular, it is reported that an implant with a narrow diameter does not cause pathological problems

Received February 9, 2021; Revised March 2, 2021; Accepted March 2, 2021

${ }^{\star}$ Corresponding author: Mee-Kyoung Son, Department of Prosthodontics, School of Dentistry, Chosun University, 303 Pilmun-daero, Donggu, Gwangju 61452, Republic of Korea.

Tel: +82-62-220-3820, Fax: +82-62-227-7811, E-mail: son0513@chosun.ac.kr

Copyright $\odot$ 2021, Oral Biology Research Institute 
even if it is placed close to the adjacent tooth within $1 \mathrm{~mm}$ [9]. In addition, the success rate of the narrow implant has been reported not only in the anterior teeth, but also in some premolar and molar areas [10].

Wilderman et al. [11] reported that a certain amount of bone resorption occurred after all mucosal gingival surgery in histological studies. Therefore, a certain amount of bone resorption was tolerated when the flap was opened and the implant was placed. Still there has been controversy whether completely covering the bone is necessary for the success of the implant or not when the flap is opened in the anterior region with a narrow bone width during implant placement. Therefore, Choi et al. [12] reported that bone resorption around the implant relatively did not occur when the implant was placed without opening the flap through animal experiments.

As with the use of Cone beam computed tomography (CBCT) in dentistry, patient information for implant surgery can be more easily converted into data in a three-dimensional way, and by using this data, virtual implant surgery and final prosthesis can be predicted in advance on the program.

In particular, in the case of using a highly accurate digital guided surgery system, it helps to place the implant in a safely planned position without opening the flap especially at the anterior site with a narrow bone width [13].

Recently, as oral scanners have become more popular and precision is increasing, various digital methods for fabrication prostheses without taking impressions and making working models have been introduced. The most popular method is to design a custom abutment using a scan body that can digitally scan instead of the conventional impression coping, or design a final prosthesis by selecting a stock abutment that can digitally scan. Another method is 'double scan methods' that readjust the margin of the custom abutment that was used during immediate loading after implantation and then, scan it two times with an oral scanner for final prosthesis design as programmed.

In this case report, implants were accurately placed in the maxillary and mandibular anterior site with narrow bone width or narrow missing space between teeth using a digital guided surgery system and narrow implant. After observation for a certain period of time after osseointegra- tion and loading, there was neither bone resorption nor pathological findings.

\section{Case}

This case report is performed for two patients who visited Yedam Dental Hospital for implant placement due to anterior teeth loss. Those two patients are healthy and don't have any medical problem. Using a digital guided surgery system (DIO Implant, Busan, Korea) exclusively for Narrow implants, a $3.3 \mathrm{~mm}$ diameter implant fixture (UFI; DIO Implant) in the maxillary anterior and a $3.0 \mathrm{~mm} \mathrm{di-}$ ameter implant fixture (UFII) in mandibular anterior teeth were placed for each patient. Periapical radiography and CBCT were taken right after implant placement, after 12 months, 24 months, and 36 months to observe the change of the alveolar bone at the implant platform area and the bone resorption around the implant fixture.

\section{Case 1}

The patient in this case is a 48-year-old female patient who visited a dental clinic with the loss of a 4-unit bridge that previously lost central incisors and connected the left and right lateral incisors. As a result of radiographic and clinical examination, a crown fracture of the right lateral incisor used as an abutment was observed (Fig. 1). Since the patient did not have a specific systemic history, the fractured right lateral incisor was extracted and 2 implants were placed in the right lateral incisor and left central incisor using a surgical guide. The alveolar bone width of the thinnest part before implant placement was $7.06 \mathrm{~mm}$ at the right lateral incisor and $5.86 \mathrm{~mm}$ at the left central incisor. It was planned to make a single crown for the left lateral incisor which was used for the abutment tooth. After the implants were placed, temporary bridge was placed at the same time.

\section{Collection of three-dimensional data from cone beam computed tomography and Intraoral scanner}

For implant placement planning and surgical guide fabrication, Intraoral scan data were collected using a Trios 2 (3shape, Copenhagen, Denmark) oral scanner, and alveolar 

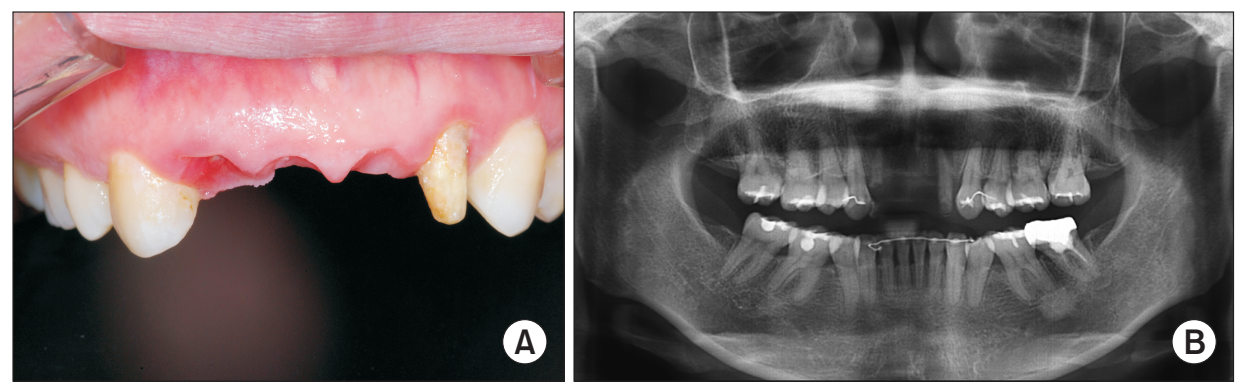

Fig. 1. (A) Intraoral view. (B) Panoramic view at the first visit.
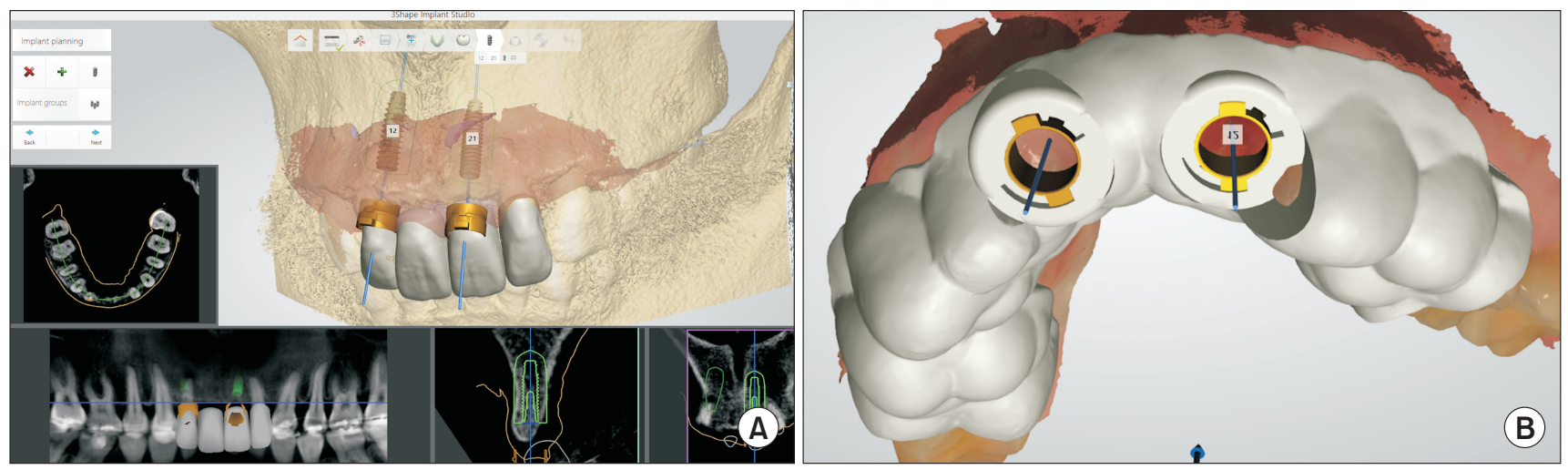

Fig. 2. (A) Determining the appropriate implant position using cone beam computed tomography and Oral scan three-dimensional data merged in the implant studio program. (B) Designed surgical guide.

bone data was taken using CBCT (PHT-30LFO; Vatech, Hwaseong, Korea). For accurate merging of scan data and $\mathrm{CBCT}$, it is necessary to acquire accurate digital impressions of the adjacent teeth to the missing area.

\section{Surgical guide, custom abutment, temporary bridge design for immediate loading}

As a result of merging the two data using the Implant studio (3Shape) program, a narrow implant of $3.3 \mathrm{~mm}$ in diameter and $13 \mathrm{~mm}$ in length (UFII) was selected for left lateral and right central teeth due to the narrow bone width and then surgical guide was designed (Fig. 2). Implant studio (3shape) data with implant placement was imported from the Dental system (3shape) program, and a custom abutment and temporary tooth were designed for immediate loading. The guide and temporary teeth designed for implant surgery were printed out using a three-dimensional (3D) printer (3D Systems, Rock Hill, SC, USA) of the MJP (MultiJet Printing; 3D Systems) method, and the custom abutment was prepared by requesting computer numerical control lathe processing (Fig. 3).

\section{Preparation of surgery site and atraumatic extraction}

On the day of surgery, the patient was rinsed intraorally with $0.12 \%$ chlorohexidine for 1 minute for oral disinfection, and 4\% lidocaine (1:100,000 adrenaline) with infiltration injection method was applied to the upper palate, alveolar crest, and labial vestibular area. If the surgical site requires extraction, a non-traumatic safe extraction procedure is needed to preserve the alveolar bone and gingiva.

Therefore, a surgical bur, a forcep dedicated to the anterior teeth, and a luxating elevator of root picker are needed. It is recommended to separate root in the mesio-distal directions to protect the bucco-lingual alveolar bone. The leaving buccal root can provide initial fixation upon immediate implantation, and can protect the buccal bone plate, which is important for aesthetics. This was called as socket shield technique [14].

\section{Drilling and Installation of fixture}

After checking the stability of the guide at the surgical site after tooth extraction, flapless implant placement surgery is performed using a narrow implant kit (UFII narrow 

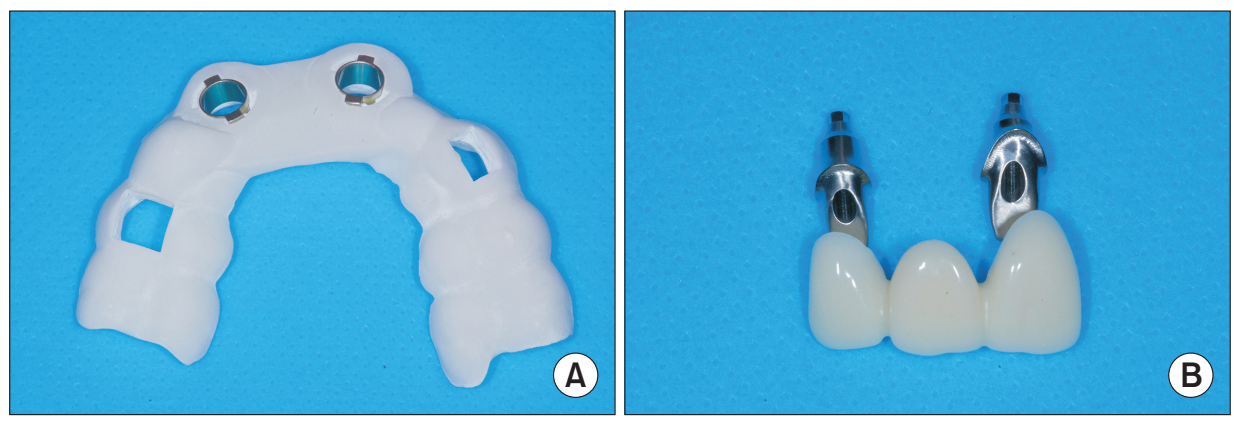

(B)
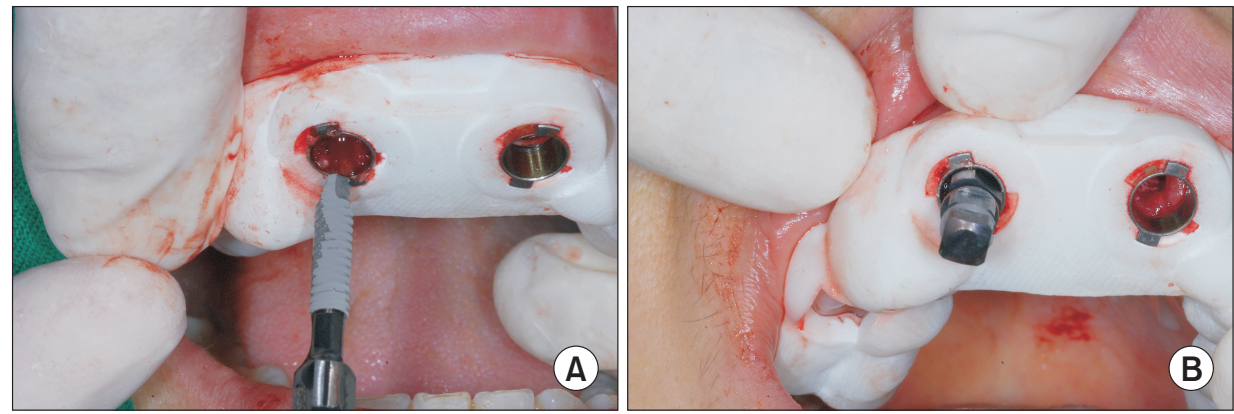

Fig. 3. (A) Surgical guides fabricated using three-dimensional printer. (B) Custom abutment and temporary teeth for immediate load.

Fig. 4. Installation of Narrow fixture (3.3 mm diameter, $13 \mathrm{~m}$ length, UFI; DIO implant, Busan, Korea). kit, DIO Implant). A predetermined $3.3 \mathrm{~mm}$ diameter, 13 $\mathrm{mm}$ length narrow fixture (UFII) was used. All drilling processes were carried out in a predetermined sequence, and a torque of less than $45 \mathrm{Ncm}$ and a drilling speed of less than $100 \mathrm{rpm}$ are recommended by the manufacturer in order to suppress heat generation while drilling. Between each drilling, sufficient normal saline irrigation was used to clean the bone particles and to cool the surgical site while drilling.

For the extracted right lateral incisor, the root area was filled with the autogenous bone removed during drilling, and then the fixture was placed. To prevent structural deformation of the fixture, an installation torque of $35 \mathrm{Ncm}$ and a speed of $30 \mathrm{rpm}$ are recommended during placement. In order to accurately connect a prefabricated abutment, it must be placed at a pre-planned height, and accurate hex alignment of the abutment and fixture is required (Fig. 4).

\section{Immediate loading with custom abutment and tem- porary crown}

As a result of measuring implant stability quotient using an Osstell mentor to check whether the installed fixture can be immediately loaded, the right lateral incisor and left central incisor showed 70 and 75 values, respectively. Therefore, the temporary teeth were connected using a pre-made custom abutment. When connecting a prefabricated custom abutment, it can be fastened using a prefabricated jig or temporary tooth, in order to prevent the rotation of the pre-placed fixture and secure it easily. Fixture and custom abutment were fastened to $20 \mathrm{Ncm}$ or less using a torque wrench to prevent fracture of the connecting screw. The color of the temporary tooth was adjusted using composite resin for the aesthetic needs of the patient (Fig. 5). Temporary teeth were formed with underocclusion and were discluded when guided laterally and anteriorly so as not to be exposed to excessive loads. The patient was recommended to eat soft and liquid food for 2 to 3 months while osseointegration was completed. After surgery, the panoramic and standardized periapical radiographs and CBCT were taking (Fig. 6).

\section{Intraoral scanbody scanning for final prosthesis}

After the bone integration was completed, the final prosthesis was designed and manufactured using an oral scanner (Trios 2; 3shape) and a scanbody without making a conventional stone working model (Fig. 7). The final prosthesis designed on the new custom abutment was made by milling zirconia blocks, and placed on the patient with an aesthetic appearance through a sintering and coloring pro- 

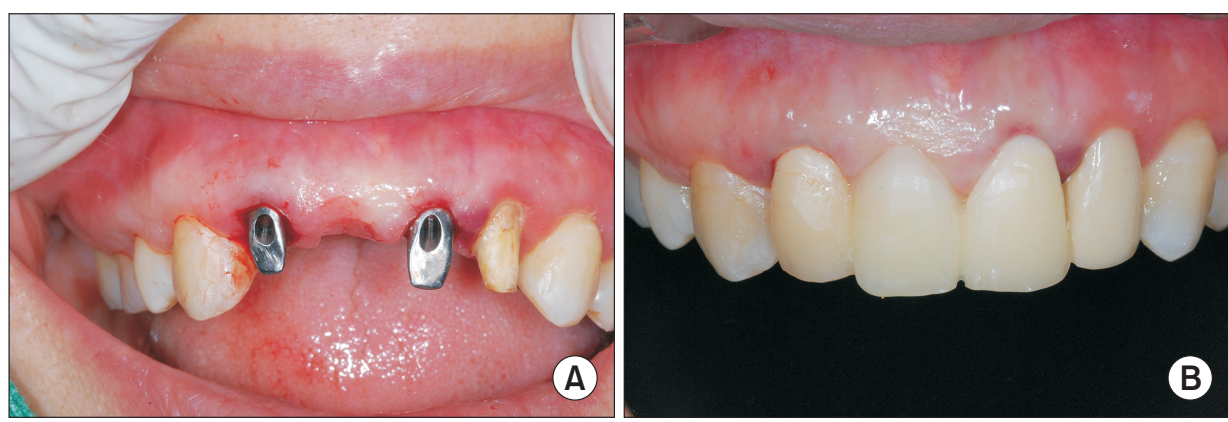

Fig. 5. The intraoral photo on the day of surgery. (A) The custom abutment and temporary teeth were connected immediately after implantation. (B) Composite resin was added on the temporary teeth to maintain the aesthetic appearance.
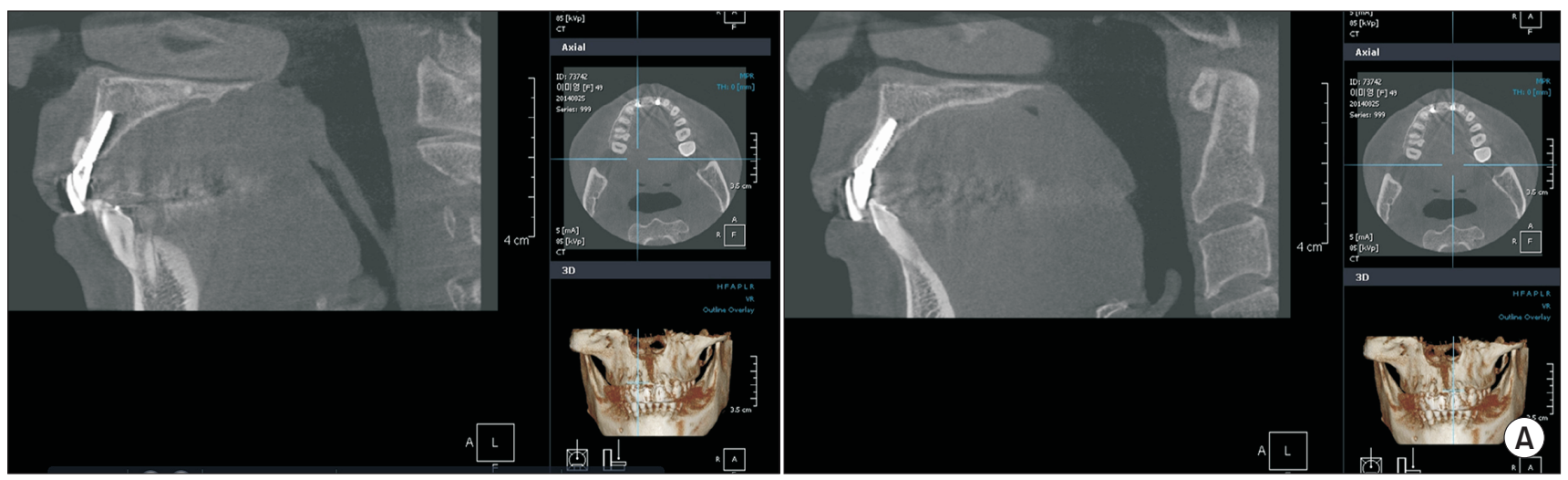

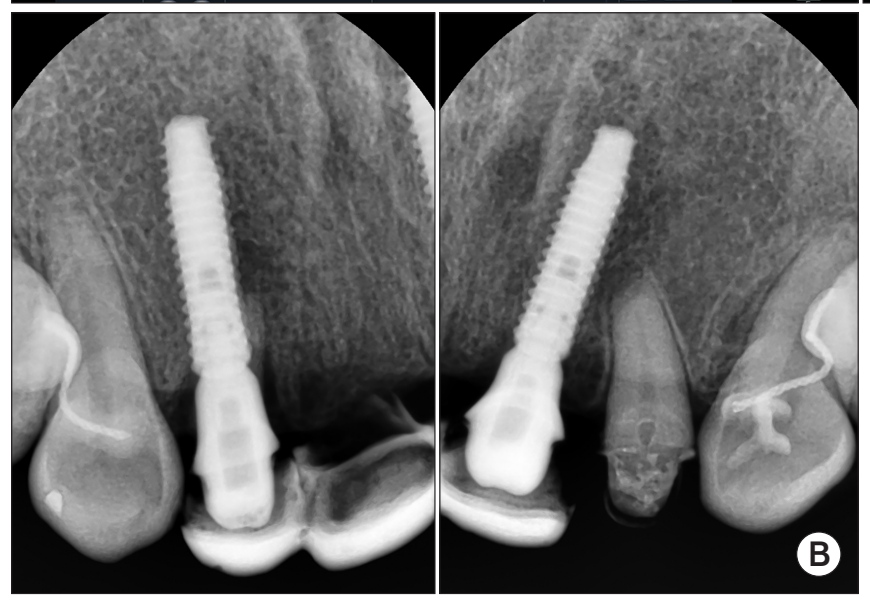

cess (Fig. 8).

\section{Evaluation of marginal bone resorption and follow up}

The standardized periapical radiograph, which was taken immediately, 12 months, 24 months, and 36 months after implantation, was converted into a high-resolution JPEG file. Using the view rulers of the photoshop program, the distance from the implant shoulder to the first bone to implant contact was measured, and the degree and the change of marginal bone resorption were observed. As a result, the periphery of the upper right lateral incisor implant
Fig. 6. (A) On cone beam computed tomography imaging, it can be seen that residual bones of less than $1 \mathrm{~mm}$ exist around fixture. (B) Periapical radiograph.

placed immediately after extraction showed an average of $0.30 \mathrm{~mm}$ marginal resorption, and the upper periphery of the left central incisor implant placed in the healed normal bone showed an average of $0.2 \mathrm{~mm}$ of marginal bone resorption (Fig. 9). On the other hand, the remaining bone surrounding the center of the fixture did not show any absorption patterns on the CBCT and was well maintained (Fig. 10). 

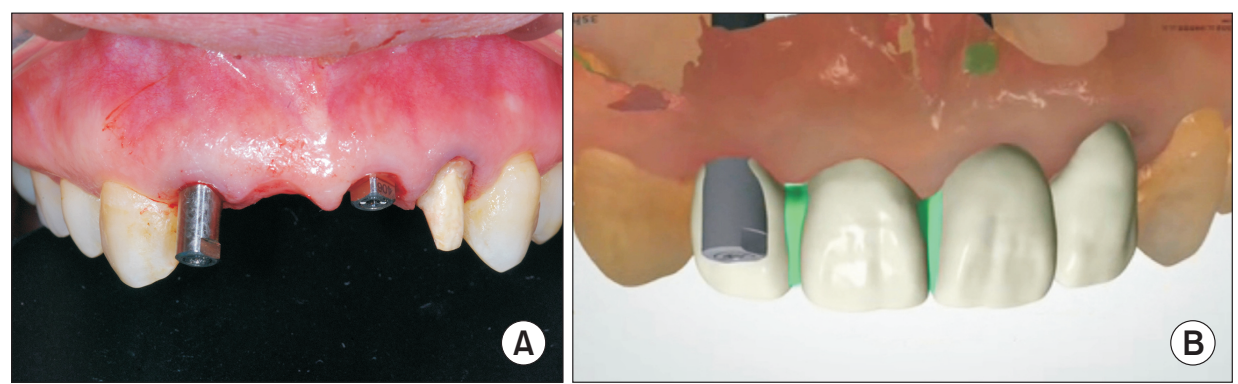

Fig. 7. (A) The scanbody connected to the fixture. Oral scan data transmitted to the dental system (3shape) and a new custom abutment and a final prosthesis were designed (B).
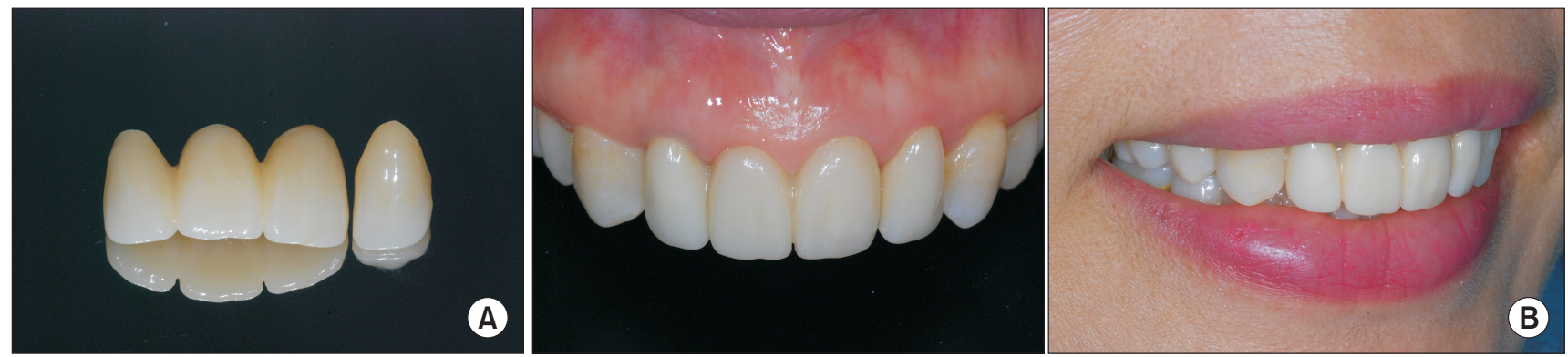

Fig. 8. Final zirconia prostheses (A) and esthetic appearance in patient's mouth (B).

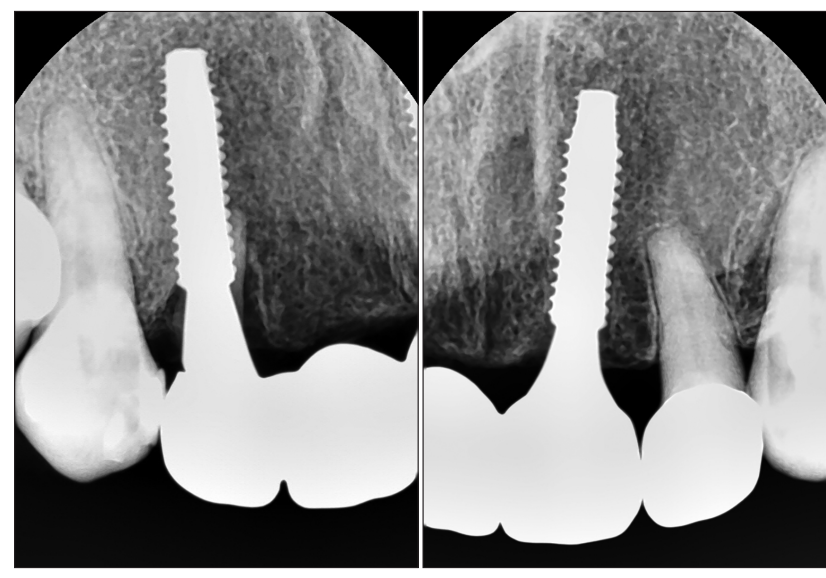

Fig. 9. The periapical view taken 24 months after the surgery. The implant is well maintained without additional bone resorption.

\section{Case2}

A 52-year-old female patient visited with the dental hospital for implant treatment due to tooth loss of \#42 and the severe mobility of the remaining mandibular anterior teeth. In the radiographic and clinical examination, alveolar bone resorption of the remaining mandibular anterior teeth (\#31, $32,41)$ was observed, and it was determined that it was impossible to maintain, and extraction was decided. The patient had a lot of fear of the dental procedure, and wor- ried about the long treatment time and aesthetic problems after the extraction and implant procedure. With the consent of the patient, implants were placed in both mandibular lateral incisors using a surgical guide at the same time as the remaining teeth were extracted and a bridge-type temporary prosthesis was immediately installed. The alveolar bone width of the thinnest part was $5.5 \mathrm{~mm}$ at \#32 and 4.5 $\mathrm{mm}$ at $\# 42$ missing area.

\section{Diagnosis on Implant studio(3shape) and flapless implant surgery with digitally guided system}

For the design of the surgical guide, the obtained CBCT data and the oral data using the Trios 3(3shape) oral scanner were merged in Implant studio program (3shape). As a result, it was confirmed that the remaining buccal-lingual bone at the center of the implant placement turned very narrow, and even if the narrow diameter of $3.0 \mathrm{~mm}$ and the length of $13 \mathrm{~mm}$ fixture (UFI) were selected and placed, the exposure of some threads was predicted (Fig. 11).

As a result of analyzing the patient's left and right canine periphery and the remaining bone at the surgical site through radiographic data and $\mathrm{CBCT}$, no periapical lesions or pathological bone pattern were observed, so it was decided to proceed as planned. It was thus decided to pro- 

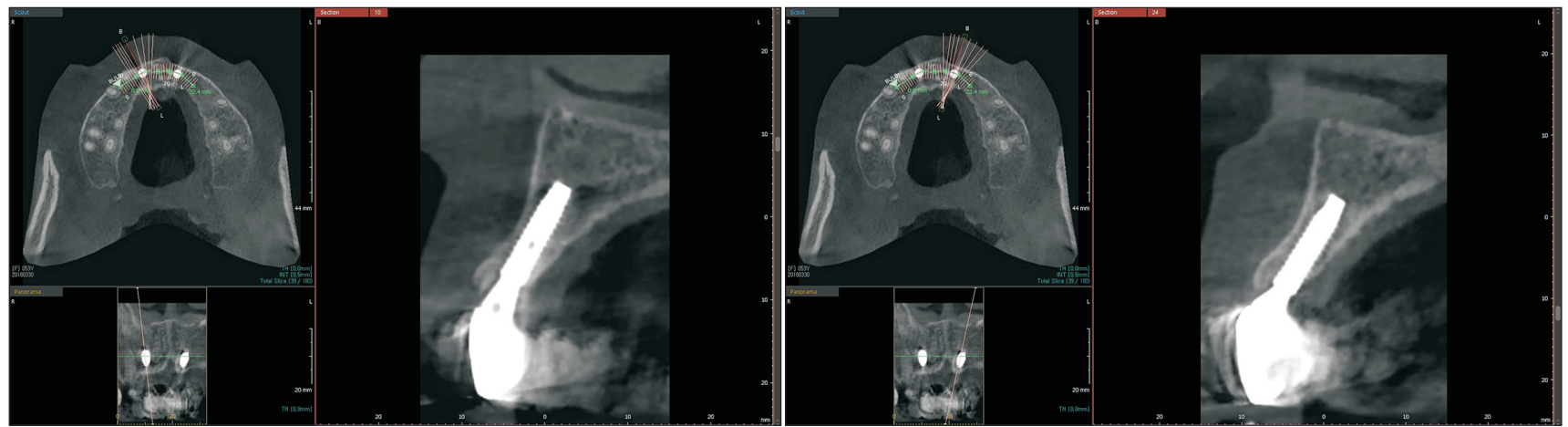

Fig. 10. From cone beam computed tomography taken 36 months after the operation, the central part of the fixture was well maintained without resorption.

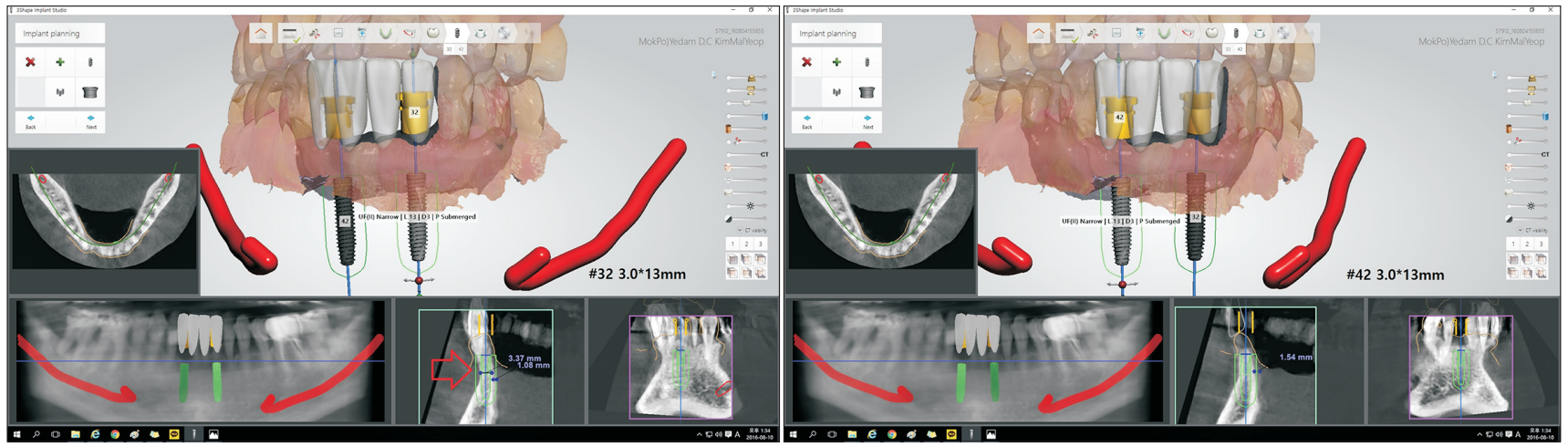

Fig. 11. Merging the cone beam computed tomography data and the oral scan data in the implant studio (3shape). A part of fixture thread at the labial side could be exposed without surrounding bone.

ceed with flapless implant surgery, but if swelling or pain occurs due to exposure of some fixture thread after surgery, the patient agreed that re-operation with bone graft may be necessary with the flap opened.

Surgical guide, custom abutment, and temporary tooth fabrication proceeded in the same manner as in case 1, and a $3.0 \mathrm{~mm}$ diameter, $13 \mathrm{~mm}$ length narrow fixture (UFII) was placed in both lateral incisors using the prepared surgical guide after tooth extraction.

After implantation, the initial implant stability measurement using the Osstell mentor showed a value of over 75 , so the pre-made custom abutment was connected to the fixture and the temporary bridge were placed (Fig. 12).

On the postoperatively taken $\mathrm{CBCT}$, the fixtures positioned as planned and the central part of some fixtures were shown as if they were exposed without residual bone (Fig. 13).

\section{Digital final zirconia prosthesis using 'Double scan methods'}

The patient did not have any symptoms of pain or swelling during osseointegration, so the final prosthesis was fabricated after 2 months. The margin of custom abutment used for immediate loading is often exposed over the gingiva after osseointegration. The same exposure has been made in this case. Instead of creating a new custom abutment, this custom abutment reused with an oral scanner digitally. The margin of the custom abutment was readjusted outside the oral cavity and it was scanned outside. After repositioned it to the fixture and the full arch and occlusion was scanned by oral scanner. By sending these two data to the dental system (3shape), that matches each other can be used to design for a prosthesis while digitally checking the prosthesis margins in the subgingival area. We called this was "double scan method".

With this method, the custom abutment used for tempo- 

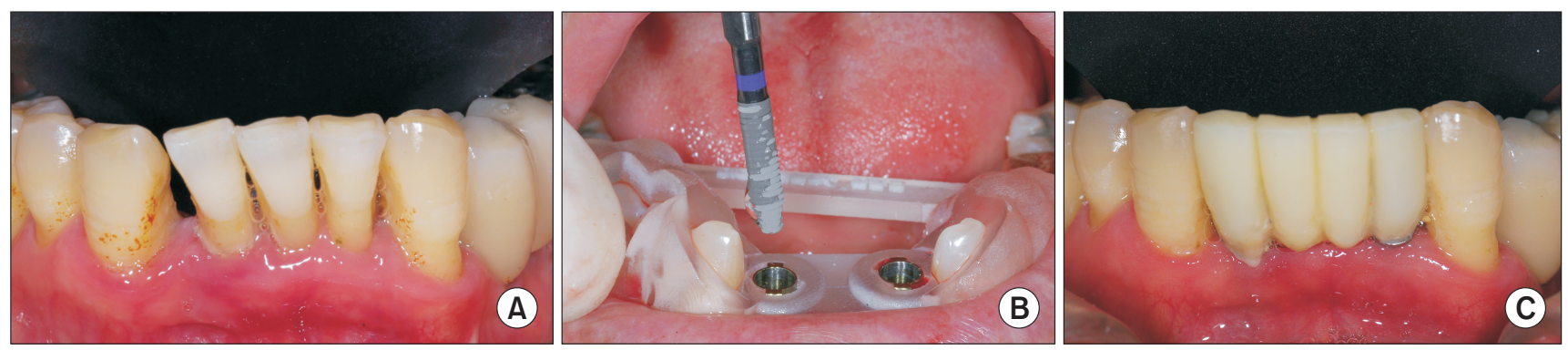

Fig. 12. (A) Intraoral view at the first visit. (B) Narrow implant (3.0 mm diameter, $13 \mathrm{~mm}$ length fixture, UFI; DIO Implant, Busan, Korea) placement with surgical guide after remaining teeth extraction. (C) Placing the custom abutment and temporary prosthesis for immediate loading.
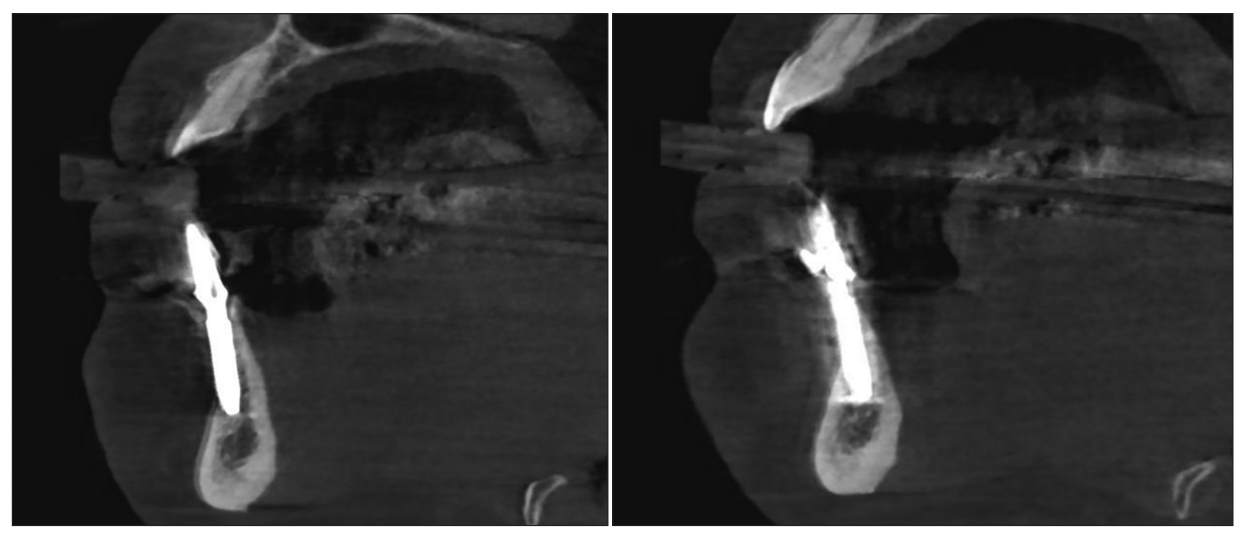

Fig. 13. On the postoperative cone beam computed tomography image, it can be seen that the labial surface of some fixtures was exposed without surrounding residual bone.

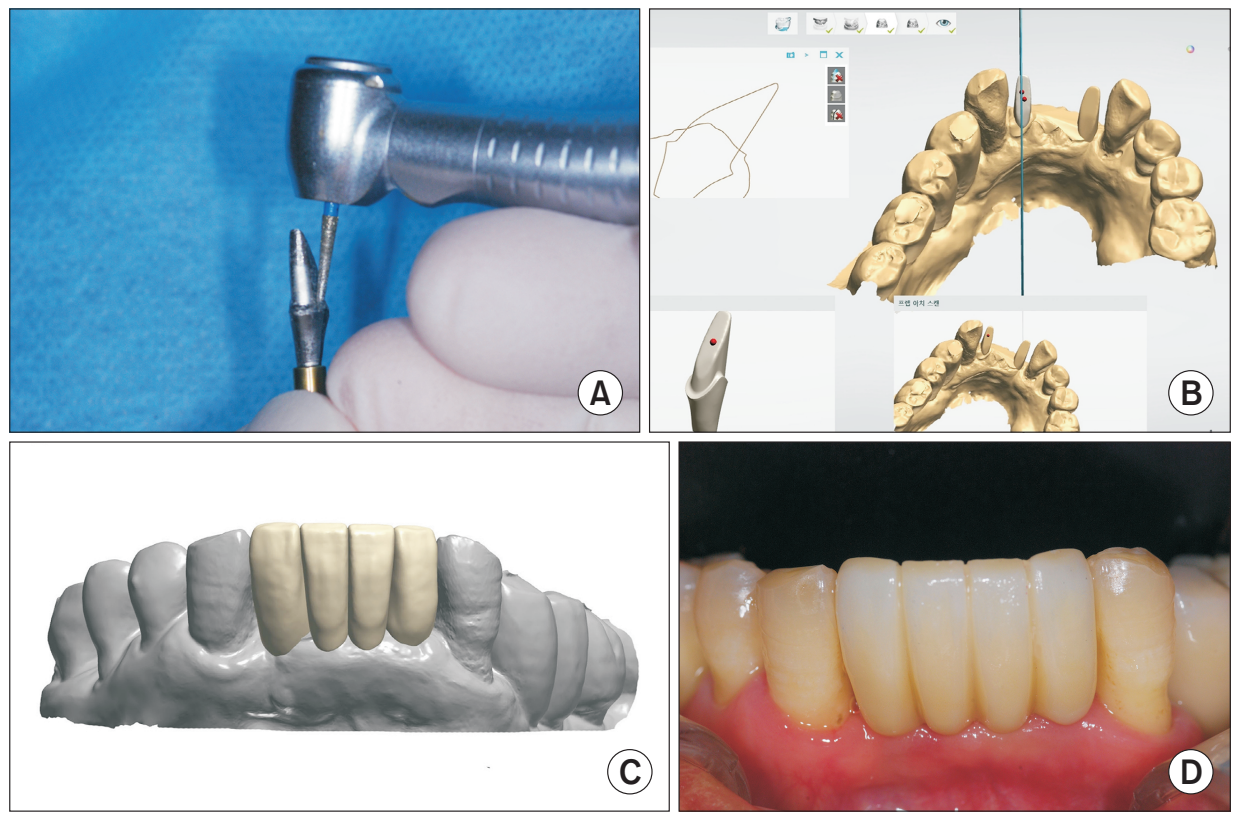

Fig. 14. (A) Readjusting the margins of the custom abutment from the outside. (B) Each readjusted custom abutment data from inside and outside of oral cavity was matching in Dental system (3shape). (C) Designed final prosthesis in software. (D) Fabricated zirconia final prosthesis. rary teeth could be readjusted and used for the final prosthesis, and it was possible to manufacture the final zirconia prosthesis without making a working model (Fig. 14).

The final prosthesis went through a temporary attach- ment for 1 month adaptation period, and then finally attached. The patient continued to be observed for 2 years at 6 months intervals from the implant placement operation. Apical radiographs and CBCT were taken at 12 and 24 

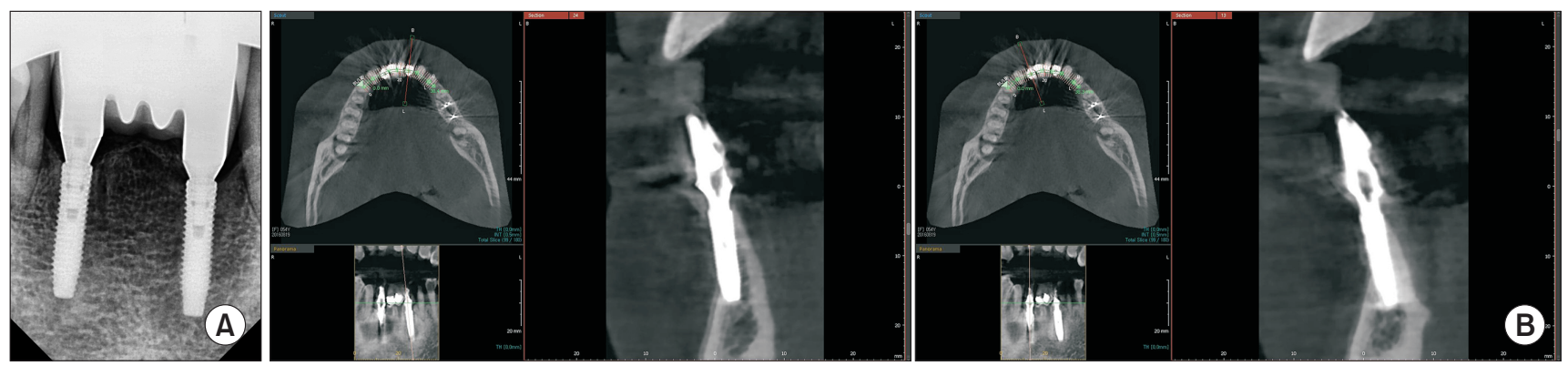

Fig. 15. (A) Apical radiographs taken 24 months postoperatively. (B) On cone beam computed tomography, the exposed part of the fixture well maintained without further bone loss and pathological findings.

months postoperatively.

A 12 months postoperatively, the bone around the implant platform showed an average of $0.35 \mathrm{~mm}$ of marginal bone resorption, but no further resorption proceeded. In $\mathrm{CBCT}$, the exposed area without residual bone around the fixture was well maintained for 12 months and 24 months without further bone loss and pathological findings (Fig. 15).

\section{Discussion}

When surgery is performed with the flap open to place an implant in the anterior region with a narrow bone width, bone graft is often accompanied by a small amount of residual bone around the fixture platform or in the center of the fixture. In some cases, if the bone graft is not performed, the threads of implant fixture are exposed during osseointegration, resulting in swelling or inflammation, which may cause implant failure.

However, there have been a lot of controversies over whether the complete bone covering is an essential factor for the success of the implant when the thread is partially exposed on the labial or buccal side after implant placement.

Ramusson et al. [15] reported that even if the screw thread was covered with bone grafts and barrier membranes, the regenerated bone hardly achieves osseointegration with the implant, and that it hardly contributes to implant stability.

In addition, Lekholm et al. [16] reported that even if the implant thread is exposed to the outside of the bone, it does not cause bone loss unless it causes problems with the health of the mucous membrane.
Choi et al. [12] reported that the bone resorption around the implant relatively did not occur when the implant was placed without opening the flap through animal experiment.

In case 1 of this paper, only remaining bones of less than $2 \mathrm{~mm}$ in the buccal and lingual sides of the fixture were present after implantation, but it was found that the remaining bones were well maintained without any change in CBCT images taken at 12, 24, and 36 months postoperatively.

As the result of Choi et al. [12], since the operation was performed without opening the flap, it is thought that even if there were few remaining bones around implant fixture, it would have been well maintained without bone resorption.

In the second case, after the implant placement, part of the fixture thread was exposed in the labial area and immediately contacted the periosteum without surrounding bone. However, as a result of CBCT examination taken 12 and 24 months postoperatively, it was well maintained without additional bone loss or pathological progression.

As in this case, if there are no pathological signs of the periphery or bone and mucous membranes around the surgical site, it is believed that even if the surface of a partially exposed fixture comes into contact with the healthy periosteum, as reported by Lekholm et al. [16], the fixture can be safely osseointegrated.

In order to place an implant without opening the flap, it is important to recognize the anatomical positional relationship such as the bone width of the surgical part before surgery. In recent years, as the performance of dental digital equipment has been improved, using a diagnosis and 
design program, the position, diameter, and length of the implant to be placed before surgery can be determined in advance, and virtual placement can be performed in advance at the desired location. This method was introduced in the field of implant treatment under the name of "Navigation system' in the early 2000s, and various digital guided surgery systems have been introduced with much research and effort to date. In addition, this method of using the navigation not only shortens the operation time by immediately applying the load using the pre-made custom abutment and temporary teeth, but also provides psychological satisfaction to the patient by solving unaesthetic problems caused by tooth loss at the same time as the surgery.

Implants with a diameter of less than $3.0 \mathrm{~mm}$ are generally manufactured as a single-piece for reasons of manufacturing process. Therefore, if it is used on the maxillary anterior teeth, since the placement direction and the prosthetic direction are different, the amount of preparation of the abutment increases, which causes frequent dropout due to insufficient retention of the prosthesis. Since the diameter of the abutment is small, the phenomenon that the implant fixture is bent due to the occlusal force has been reported by experiments [17].

Recently, with the development of implant processing technology and surface treatment technology, for fixtures with a diameter of $3.0 \mathrm{~mm}$ to $3.5 \mathrm{~mm}$ or less, a narrow implant in which a fixture and abutment are separately manufactured has been developed and applied to clinical applications.

Two pieces implants have the advantage of using an angled stock abutment or a custom abutment when the angle of implant placement and the prosthetic angle do not match. Besides it has the advantage of being able to take digital impressions using a scan body and oral scanner.

The two pieces Narrow implant has a thin implant platform that surrounds the abutment is thin. Therefore, the manufacturers recommended to tighten the insertion torque of $35 \mathrm{Ncm}$ or less for the fixture and $20 \mathrm{Ncm}$ or less when fastening the abutment to prevent tearing on the upper part of implant fixture and screw fracture.

Cristian et al. [18] placed narrow implants at the extraction and healed sites, and simultaneously performed immediate loading. As a result, it was reported that there was a change within $0.5 \mathrm{~mm}$ in all observations for 2 years. This case reports that a patient who was placed with a narrow implant in the anterior region with a narrow bone width was applied with an immediate load. The patient's bone changes around the implant were observed for 2 to 3 years, and the average bone resorption was $0.3 \mathrm{~mm}$ in the case of immediate placement in the extraction socket. In the case of implantation in the healed bone, the average bone resorption was $0.2 \mathrm{~mm}$. Therefore, it was observed that all bone resorption results were within $0.5 \mathrm{~mm}$.

In both cases of this report, bone graft was not performed because the gap between the implant platform and the adjacent bone of the healing socket is within the jumping distance [19], which allows the bone to be naturally filled after placement.

With the introduction of the digital guided surgery system, the most worrisome was the error that appeared before and after surgery, and various results using various systems were reported. Analyzing the average errors of the existing literature published on this problem, the average of $1.22 \mathrm{~mm}$ in the implant platform part, $1.51 \mathrm{~mm}$ in the apex part, and the average angular deviation was 4.9 degrees [20-23].

Therefore, many attempts have been introduced to reduce the error of the digital guided surgery system and to increase the precision. Karim et al. [24] reported that the precision can be improved by reducing the drilling distance coming out of the sleeve by adjusting the length of the sleeve or shortening the length of the implant.

Choi et al. [12] used a digital guided surgery system (DIO Implant) dedicated to narrow implants, including a long tube that accurately guides a long drill for accurate narrow implant placement, and placed 40 narrow implants on the anterior region of 32 patients. As a result of comparing the errors, an average linear deviation of $0.46 \mathrm{~mm}$ from the implant platform, $0.67 \mathrm{~mm}$ from the implant apex, and an angular deviation of 1.40 degrees were reported.

When using a digital guided surgery system, heat may be generated during the drilling process, and bone necrosis may occur after surgery. Therefore, in this case, heat generation was prevented by repeatedly using all drilling at $100 \mathrm{rpm}$ or less under sufficient normal saline irrigation. In addition, a long drill and a long tube were used to increase 
the precision of the guided surgery system, and a pre-made custom abutment and a polymethylmethacrylate temporary crown were used for immediate loading.

In the previous studies, several authors reported that the use of narrow-diameter implants increased the risk of prosthetic complications or implant fracture [8,25]. This phenomenon was not observed in this case, and it is expected that this problem will be gradually resolved with the development of implant materials or systems. Narrow implants are still very limited in clinical use.

However, if an implant can be placed through a minimally invasive method and maintained for a long period of time for patients whose bone graft or additional surgery could not performed, it can be a very useful option in clinical practice.

In addition to this case report, it is believed that a larger number of patients and longer follow-up will be needed to confirm the same results.

In conclusion, using 3D data and a digital guided surgery system, a narrow diameter implant can be placed in an exact position with flapless surgery. The patient has the advantage of reducing postoperative pain and obtaining a sense of aesthetic stability with an immediate-loaded temporary tooth. In addition, it was possible to produce the prosthetic process in a convenient digital method without a working model.

After the implant placement, even if minimal residual bone remained around the fixture or some threads were exposed outside the bone and touched the healthy periosteum, it was found that it remained stable without bone resorption for over 24 months.

However, the use of narrow-diameter implants should be limited to the maxillary and mandibular anterior teeth where the occlusal load is relatively low, and it is considered that it is necessary to suggest an optional criterion for use through more cases.

\section{Acknowledgements}

This study was supported by a research fund from Chosun University Dental Hospital, 2020.

\section{Conflicts of Interest}

The authors declare that they have no competing interests.

\section{ORCID}

\author{
Jae-Seok Kang \\ https://orcid.org/0000-0003-0419-2559 \\ Seon-A Kim \\ https://orcid.org/0000-0002-0942-6046 \\ Yu-Ri Heo \\ https://orcid.org/0000-0002-1806-3822 \\ Mee-Kyoung Son \\ https://orcid.org/0000-0001-9225-1744
}

\section{References}

1. Pham AN, Fiorellini JP, Paquette D, Williams RC, Weber HP. Longitudinal radiographic study of crestal bone levels adjacent to non-submerged dental implants. J Oral Implantol 1994;20:26-34.

2. Hermann JS, Cochran DL, Nummikoski PV, Buser D. Crestal bone changes around titanium implants. A radiographic evaluation of unloaded nonsubmerged and submerged implants in the canine mandible. J Periodontol 1997;68:11171130. doi: 10.1902/jop.1997.68.11.1117.

3. Spray JR, Black CG, Morris HF, Ochi S. The influence of bone thickness on facial marginal bone response: stage 1 placement through stage 2 uncovering. Ann Periodontol 2000;5:119-128. doi: 10.1902/annals.2000.5.1.119.

4. Yang G, Chen L, Gao Y, Liu H, Dong H, Mou Y. Risk factors and reoperative survival rate of failed narrow-diameter implants in the maxillary anterior region. Clin Implant Dent Relat Res 2020;22:29-41. doi: 10.1111/cid.12867.

5. Allum SR, Tomlinson RA, Joshi R. The impact of loads on standard diameter, small diameter and mini implants: a comparative laboratory study. Clin Oral Implants Res 2008;19:553-559. doi: 10.1111/j.1600-0501.2007.01395.x.

6. Al-Johany SS, Al Amri MD, Alsaeed S, Alalola B. Dental implant length and diameter: a proposed classification scheme. J Prosthodont 2017;26:252-260. doi: 10.1111/ jopr.12517.

7. Schiegnitz E, Al-Nawas B. Narrow-diameter implants: a systematic review and meta-analysis. Clin Oral Implants Res 2018;29 Suppl 16:21-40. doi: 10.1111/clr.13272.

8. Zinsli B, Sägesser T, Mericske E, Mericske-Stern R. Clinical evaluation of small-diameter ITI implants: a prospective study. Int J Oral Maxillofac Implants 2004;19:92-99.

9. Trbakovic A, Bongenhielm U, Thor A. A clinical and ra- 
diological long-term follow-up study of narrow diameter implants in the aesthetic area. Clin Implant Dent Relat Res 2018;20:598-605. doi: 10.1111/cid.12627.

10. Alrabiah M, Al Deeb M, Alsahhaf A, AlFawaz YF, Al-Aali KA, Vohra F, Abduljabbar T. Clinical and radiographic assessment of narrow-diameter and regular-diameter implants in the anterior and posterior jaw: 2 to 6 years of follow-up. J Periodontal Implant Sci 2020;50:97-105. doi: 10.5051/jpis.2020.50.2.97.

11. Wilderman MN, Wentz FM, Orban BJ. Histogenesis of repair after mucogingival surgery. J Periodontol 160;31:283299. doi: 10.1902/jop.1960.31.4.283.

12. You TM, Choi BH, Li J, Xuan F, Jeong SM, Jang SO. Morphogenesis of the peri-implant mucosa: a comparison between flap and flapless procedures in the canine mandible. Oral Surg Oral Med Oral Pathol Oral Radiol Endod 2009;107:66-70. doi: 10.1016/j.tripleo.2008.05.045.

13. Fang Y, An X, Jeong SM, Choi BH. Accuracy of computerguided implant placement in anterior regions. J Prosthet Dent 2019;121:836-842. doi: 10.1016/j.prosdent. 2018.07.015.

14. Hürzeler MB, Zuhr O, Schupbach P, Rebele SF, Emmanouilidis N, Fickl S. The socket-shield technique: a proof-ofprinciple report. J Clin Periodontol 2010;37:855-862. doi: 10.1111/j.1600-051X.2010.01595.x.

15. Rasmusson L, Sennerby L, Lundgren D, Nyman S. Morphological and dimensional changes after barrier removal in bone formed beyond the skeletal borders at titanium implants. A kinetic study in the rabbit tibia. Clin Oral Implants Res 1997;8:103-116. doi: 10.1034/j.1600-0501.1997. 080205.x.

16. Lekholm U, Sennerby L, Roos J, Becker W. Soft tissue and marginal bone conditions at osseointegrated implants that have exposed threads: a 5-year retrospective study. Int J Oral Maxillofac Implants 1996;11:599-604.

17. Tuzzolo Neto H, Tuzita AS, Gehrke SA, de Vasconcellos Moura R, Zaffalon Casati M, Mikail Melo Mesquita A. A comparative analysis of implants presenting different diameters: extra-narrow, narrow and conventional. Materials
(Basel) 2020;13:1888. doi: 10.3390/ma13081888.

18. Peron C, Romanos G. Immediate provisionalization of single narrow implants in fresh extraction sockets and healed sites: clinical and radiographic outcomes of 2 years followup. Int J Periodontics Restorative Dent 2020;40:417-424. doi: 10.11607/prd.4622.

19. Botticelli D, Berglundh T, Buser D, Lindhe J. The jumping distance revisited: an experimental study in the dog. Clin Oral Implants Res 2003;14:35-42. doi: 10.1034/j.16000501.2003.140105.x.

20. Zhou W, Liu Z, Song L, Kuo CL, Shafer DM. Clinical factors affecting the accuracy of guided implant surgery-a systematic review and meta-analysis. J Evid Based Dent Pract 2018;18:28-40. doi: 10.1016/j.jebdp.2017.07.007.

21. Bover-Ramos F, Viña-Almunia J, Cervera-Ballester J, Peñarrocha-Diago M, García-Mira B. Accuracy of implant placement with computer-guided surgery: a systematic review and meta-analysis comparing cadaver, clinical, and in vitro studies. Int J Oral Maxillofac Implants 2018;33:101115. doi: 10.11607/jomi.5556.

22. Tahmaseb A, Wismeijer D, Coucke W, Derksen W. Computer technology applications in surgical implant dentistry: a systematic review. Int J Oral Maxillofac Implants 2014;29 Suppl:25-42. doi: 10.11607/jomi.2014suppl.g1.2.

23. Lin CC, Wu CZ, Huang MS, Huang CF, Cheng HC, Wang DP. Fully digital workflow for planning static guided implant surgery: a prospective accuracy study. J Clin Med 2020;9:980. doi: 10.3390/jcm9040980.

24. El Kholy K, Janner SFM, Schimmel M, Buser D. The influence of guided sleeve height, drilling distance, and drilling key length on the accuracy of static Computer-Assisted Implant Surgery. Clin Implant Dent Relat Res 2019;21:101107. doi: 10.1111/cid.12705.

25. Romeo E, Lops D, Amorfini L, Chiapasco M, Ghisolfi M, Vogel G. Clinical and radiographic evaluation of smalldiameter (3.3- $\mathrm{mm}$ ) implants followed for 1-7 years: a longitudinal study. Clin Oral Implants Res 2006;17:139-148. doi: 10.1111/j.1600-0501.2005.01191.x. 